\title{
PREVALENCIA, SEVERIDAD Y NECESIDAD DE TRATAMIENTO DE LA ENFERMEDAD PERIODONTAL EN PERSONAL DEL BATALLÓN DE ARTILLERÍA DE DEFENSA AÉREA NO. 2 NUEVA GRANADA
}

\author{
${ }^{1}$ Claudia Liliana Álvarez Cortés, ${ }^{2}$ Vietnamila Rico Jaimes, ${ }^{3}$ Juan Carlos Gómez Herrera \\ ${ }^{1}$ Estudiante X Semestre F. de Odontología U. Santo Tomás, Colombia. \\ ${ }^{2}$ Odontóloga U. Santo Tomás, Especialista en Periodoncia U Cooperativa de Colombia, Docente U. Santo Tomás, Colombia. \\ ${ }^{3}$ Odontólogo U. Santo Tomás, Especialista en Rehabilitación Oral U. Santo Tomás, Docente U. Santo Tomás, Colombia.
}

Autor responsable de correspondencia: Claudia Liliana Álvarez Cortés

Correo electrónico: clalvarez_3@hotmail.com

\begin{abstract}
RESUMEN
Objetivo: determinar la prevalencia, severidad y la necesidad de tratamiento de la enfermedad periodontal en soldados pertenecientes al Batallón de Artillería y Defensa Aérea No. 2 Nueva Granada de la ciudad de Barrancabermeja.

Materiales y métodos: se realizó un estudio de corte transversal. El universo estuvo compuesto por una unidad táctica del batallón Nueva Granada. Se realizó un muestreo no probabilístico por conveniencia y se incluyeron 120 soldados para el estudio. Para determinar el estado de salud periodontal se utilizó un periodontograma y el Índice Comunitario de Necesidades de Tratamiento Periodontal propuesto por la Organización Mundial de la Salud aplicados por el mismo investigador previamente capacitado y calibrado. Se calcularon frecuencias y porcentajes para las variables categóricas y medidas de tendencia central y de dispersión para las variables cuantitativas. Se realizó la prueba $\mathrm{Chi}^{2}$ con un valor de $\mathrm{p}<0,05$ considerado estadísticamente significativo.

Resultados: se encontró una prevalencia de enfermedad periodontal del 100\%. No se observó una diferencia estadísticamente significativa entre la enfermedad periodontal y las variables analizadas (edad, estrato socioeconómico, nivel de escolaridad). La severidad de la enfermedad fue del 74,8\% para los niveles leve, moderada y severa. El Índice Comunitario de Necesidad de Tratamiento Periodontal fue representativo con el criterio 3 (bolsas de $4 \mathrm{~mm}-5 \mathrm{~mm}$ ) con un 34,6\% y la Necesidad de Tratamiento requerida fue Instrucción en Higiene bucal, profilaxis y alisado radicular a campo cerrado.

Conclusiones: la prevalencia, severidad y necesidad de tratamiento observadas fueron altas. Todos los soldados del batallón Nueva Granada evaluados requerían tratamiento. Es necesario encaminar esfuerzos con el fin de mejorar las condiciones de salud bucal y periodontal en esta población. [Álvarez CL, Rico V, Gómez JC. Prevalencia, severidad y necesidad de tratamiento de la enfermedad periodontal en personal del Batallón de Artillería de Defensa Aérea No. 2 Nueva Granada. Ustasalud 2013; $12: 11$ - 19]
\end{abstract}

Palabras clave: Prevalencia, Gingivitis, Periodontitis, Personal Militar.

\section{PREVALENCE, SEVERITY AND PERIODONTAL TREATMENT NEEDS OF PERIODONTAL DISEASE IN AIR DEFENSE ARTILLERY BRANCH \# 2 NUEVA GRANADA}

\begin{abstract}
Objective: to assess the prevalence, severity and periodontal treatment needs in soldiers from the Air Defense Artillery Branch \# 2 Nueva Granada in the city of Barrancabermeja.

Methods: a cross sectional study was done. A tactical unit of the Nueva Granada Battalion was considered as the universe. A nonprobability convenience sampling was conducted and 120 soldiers were included. To determine the status of periodontal health a periodontal chart was proposed by the World Health Organization was used, also the Community Periodontal Index of Treatment Needs was measured. Frequencies and percentages for categorical variables and measures of central tendency and dispersion for quantitative variables were calculated. The $\mathrm{Chi}^{2}$ test was performed with a value of $\mathrm{p}<0.05$ considered statistically significant.

Results: the prevalence of periodontal disease found was $100 \%$. No statistically significant difference between periodontal disease and the analyzed variables (age, socioeconomic status, educational level) were observed. The severity of the disease was $74.8 \%$ for the mild, moderate and severe levels. The Community Periodontal Index of Treatment Need was representative in criterion 3 (pockets $4 \mathrm{~mm}-$ $5 \mathrm{~mm}$ ) with $34.6 \%$ and the need for treatment required was Oral hygiene instructions and root surface debridement.

Conclusions: the prevalence, severity and need for treatment observed were high. All the soldier evaluated from the Granada Battalion required treatment. It is necessary to direct efforts to improve the conditions of oral and periodontal health in this population.
\end{abstract}

Key words: Prevalence, Gingivitis, Periodontits, Military personnel.

Recibido para publicación: junio 8 de 2013. Aceptado para publicación: junio 22 de 2013. 


\section{INTRODUCCIÓN}

La enfermedad periodontal es considerada una enfermedad crónica, infecciosa-inflamatoria que puede llevar a la pérdida total de los tejidos de soporte del diente. ${ }^{1}$ Además, es considerada por la Organización Mundial de la Salud (OMS) como uno de los mayores problemas globales que afectan la salud bucal en países industrializados y que aumenta en los países en desarrollo, en especial en las comunidades menos favorecidas. ${ }^{2}$ La gingivitis y la periodontitis son las patologías que afligen a un alto porcentaje de la población, incluso a individuos jóvenes. ${ }^{2}$

Algunos factores de riesgo pueden agravar una enfermedad periodontal establecida; entre éstos se puenden citar la presencia de patologías sistémicas, el consumo de medicamentos como los esteroides, estilos de vida en los que predomine el consumo de tabaco y alcohol, altos niveles de éstres, entre otros. ${ }^{3,4}$

En el 2007, se evaluó el impacto del programa de promoción y prevención en salud oral al interior del Sistema de Salud de las Fuerza Militares y se pudo evidenciar la elevada prevalencia de caries dental y enfermedad periodontal, en especial en la población entre los 16 y 24 años. $^{5}$

Si bien es importante la ejecución de programas de promoción y prevención en todos los usuarios del sistema de salud de las fuerzas militares también es necesario que éstos sean realizados directamente con los soldados que se encuentran en las áreas de combate o fuera de la base a la cual pertenecen, pues es allí donde se incrementan las condiciones de vulnerabilidad y aumenta el grado de susceptibilidad a la enfermedad.

Conocer el estado de salud periodontal, el grado de severidad y las necesidades de tratamiento de una población resulta relevante para detectar los principales problemas desencadenantes de la enfermedad y los datos específicos sobre los requerimientos de fuerza de trabajo. Adicionalmente, estos resultados constituyen herramientas sólidas para el mejoramiento de los protocolos de diagnóstico, tratamientos preventivos y remisiones oportunas al especialista en periodoncia, lo cual permite diagnosticar y tratar a tiempo la enfermedad. Por lo tanto, el objetivo de la presente investigación fue determinar la prevalencia, severidad y necesidad de tratamiento de enfermedad periodontal en los soldados del Batallón de Artillería y Defensa Aérea No. 2 Nueva Granada de la ciudad de Barrancabermeja.

\section{MATERIALES Y MÉTODOS}

Se realizo un estudio de corte transversal. El universo lo constituyó una unidad táctica del batallón denomina- da "Batallón de Artillería de Defensa Aérea No. 2 Nueva Granada". Se realizó un muestreo no probabilístico por conveniencia y se incluyeron 120 soldados entre los 18 y 37 años de edad. Estos se encontraban sistémicamente sanos, no presentaban tratamiento de ortodoncia y tampoco estaban bajo terapía antibiótica en los últimos treinta días.

Se realizó una entrevista estructurada mediante una encuesta en la que se preguntó sobre la edad, el estrato socioeconómico de su núcleo familiar, el nivel de escolaridad, sus prácticas, hábitos y conocimientos de higiene bucal. El examen clínico fue realizado en el consultorio odontológico del dispensario del Batallón Nueva Granada durante los meses de mayo, junio y julio de 2012, para tal efecto el examinador cumplió con todas las normas y elementos de bioseguridad requeridos.

La detección de los niveles de placa dentobacteriana se realizó utilizando el índice cuantificado de placa por ser un índice rápido, eficaz y confiable. Para la evaluación periodontal, se examinaron todos los dientes con un espejo bucal No. 5 y la sonda periodontal Carolina del Norte (Hu-Friedy ${ }^{\circledR}$ ), al tomar las medidas del margen gingival y la profundidad del surco, lo que permitió hallar el nivel de inserción clínico. Todos estos datos quedaron registrados en el periodontograma que hizo parte del instrumento recolector de datos.

Previo al registro de las variables, se realizó la calibración en la toma de los índices con un Especialista en Periodoncia quien fue el referente. El coeficiente Kappa obtenido fue 0,85 . Posteriormente, se realizó una prueba piloto en donde se tomaron datos en diez pacientes en las instalaciones de la Universidad Santo Tomás.

Se determinó la severidad de la enfermedad periodontal al tener en cuenta el nivel de pérdida de inserción clínica (NIC) asi:

\begin{tabular}{|c|c|c|c|}
\hline NIC & $=1 \mathrm{~mm}-2 \mathrm{~mm}$ & $\rightarrow$ & Leve \\
\hline $\mathrm{NI}$ & $=3 \mathrm{~mm}-4 \mathrm{~mm}$ & $\rightarrow$ & Moderada \\
\hline & $=5 \mathrm{~mm}$ ó más & - & Severa \\
\hline
\end{tabular}

Los marcadores clínicos evaluados para determinar la Necesidad de Tratamiento Periodontal fueron sangrado al sondaje, cálculos, presencia de bolsas periodontales de $4 \mathrm{~mm}$ - $5 \mathrm{~mm}$ o mayor de $6 \mathrm{~mm}$, los cuales hacen parte integral del Índice Comunitario de Necesidades de Tratamiento Periodontal (CPITN).

\section{Análisis estadístico}

Para el análisis estadístico se utilizo el paquete SPSS 20 , se calcularon frecuencias y porcentajes para las variables categóricas y medidas de tendencia central y de dispersión para las variables cuantitativas. Se realizó una prueba de $\mathrm{Chi}^{2} \mathrm{y}$ se consideró un valor de $\mathrm{p}<0,05$ como estadísticamente significativo. 


\section{Consideraciones éticas}

Bajo la resolución 8430 de 1993 y en su Artículo 11 se define este tipo de estudio como de "riesgo mínimo". Se aplicaron los principios éticos de Autonomía, Beneficencia, No maleficencia y Justicia que quedaron consignados en el consentimiento informado.

\section{RESULTADOS}

Se examinaron 120 soldados que evidenciaron una prevalencia de la enfermedad periodontal del $100 \%$. Para la gingivitis, la prevalencia fue de $5,8 \%$ con un total de siete pacientes afectados y para la periodontitis, $113(94,2 \%)$ soldados tenían esta entidad lo que indicó que toda la población evaluada presentaba algun grado de compromiso periodontal.

Al relacionar la edad con la enfermedad periodontal no se encontró una diferencia estadísticamente significativa $(p=0,427)$. Para el grupo $1,5(5,1 \%)$ soldados presentaron gingivitis y $94(94,9 \%)$ soldados, periodontitis. Para el grupo 2, 2 (9,5\%) soldados presentaron gingivitis y $19(90,5 \%)$, periodontitis. En cuanto al estrato socioeconómico 1, 58 (95,1\%) soldados presentaron periodontitis y tres $(4,9 \%)$ soldados, gingivitis. De los soldados que reportaron pertenecer al estrato socioeconómico 2, 29 (90,6\%) tenían periodontitis y tres $(9,4 \%)$, gingivitis. En el estrato $3,23(95,8 \%)$ presentaron periodontitis y uno $(4,2 \%)$ gingivitis. Por último, los tres soldados incluidos en el estrato 4, presentaron periodontitis. No se encontró una diferencia estadísticamente significativa entre la presencia de enfermedad periodontal y el estrato socioeconómico $(p=0,770)$ (Tabla 1$)$.

Con respecto a la relación entre la enfermedad periodontal y el nivel de escolaridad no se encontró una diferencia estadísticamente significativa $(p=0,216)$. En la tabla 1 se observa la distribución de la edad, el estrato socioeconómico y el nivel de escolaridad con la enfermedad periodontal.

Tabla 1. Distribución de la edad, estrato y nivel de escolaridad según la enfermedad periodontal

\begin{tabular}{|c|c|c|c|}
\hline \multirow{3}{*}{ Variables } & \multicolumn{2}{|c|}{ Enfermedad Periodontal } & \multirow{3}{*}{$\mathbf{p}$} \\
\hline & Gingivitis & Periodontitis & \\
\hline & n (\%) & n (\%) & \\
\hline Edad & & & 0,427 \\
\hline Grupo 1 & $5(5,1)$ & $94(94,9)$ & \\
\hline Grupo 2 & $2(9,5)$ & $19(90,5)$ & \\
\hline Estrato socioeconómico & & & 0,770 \\
\hline Estrato 1 & $3(4,9)$ & $58(95,1)$ & \\
\hline Estrato 2 & $3(4,9)$ & $29(90,6)$ & \\
\hline Estrato 3 & $1(4,2)$ & $23(95,8)$ & \\
\hline Estrato 4 & - & $3(100,0)$ & \\
\hline Nivel de escolaridad & & & 0,216 \\
\hline Primaria & -- & $36(100,0)$ & \\
\hline Secundaria & $7(9,3)$ & $68(90,7)$ & \\
\hline Técnica & -- & $3(100,0)$ & \\
\hline Profesional & - - & $6(100,0)$ & \\
\hline
\end{tabular}

\section{Prevalencia de la enfermedad periodontal según las prácticas, uso de elementos y educación en hi- giene oral}

No se encontró una diferencia estadísticamente significativa entre el uso de enjuague bucal y la enfermedad periodontal $(p=0,567)$. Sesenta y cuatro $(53,3 \%)$ soldados reportaron no usar enjuague bucal. De éstos, $61(95,3 \%)$ presentaron periodontitis y tres $(4,7 \%)$ gingivitis. De los $56(46,7 \%)$ que si lo usaban, $52(92,9 \%)$ tenían periodontitis y cuatro $(7,1 \%)$ gingivitis (Tabla 2 ).
En relación con el uso de la seda dental se observó una diferencia estadísticamente significativa entre los grupos $(p=0,008)$. Setenta y dos $(60 \%)$ soldados reportaron no utilizar la seda dental dentro de sus prácticas de higiene; de éstos, 70 (97,2\%) presentaban periodontitis y dos $(2,8 \%)$ gingivitis. De los 48 (40\%) que si la usaban, $43(89,6 \%)$ presentaban periodontitis y cinco $(10,4 \%)$ gingivitis (Tabla 2$)$.

Con respecto a los resultados obtenidos entre la asociación del uso de palillos y la enfermedad periodontal se encontró una diferencia estadísticamente 
significativa $(\mathrm{p}=0,006)$. Cuarenta y cuatro $(36,7 \%)$ soldados refirieron no usar este elemento. De este número, $38(86,4 \%)$ presentaban periodontitis y seis $(13,6 \%)$ tenían gingivitis. Setenta y seis $(63,3 \%)$ soldados reportaron el uso de palillos y de éstos, 75 $(98,7 \%)$ presentaron periodontitis y el soldado restante $(1,3 \%)$ refirió gingivitis (Tabla 2 ).
Para la variable sangrado al cepillado no se encontró una diferencia estadísticamente significativa $(\mathrm{p}=0,206)$. Cuarenta y dos $(35,0 \%)$ soldados no presentaban sangrado, de este grupo, $38(90,5 \%)$ presentaban periodontitis y cuatro $(9,5 \%)$ gingivitis. Los demás resultados en relación con las variables registradas se encuentran en la Tabla 2.

Tabla 2. Distribución de prácticas, uso de elementos e instrucción en higiene oral según la condición periodontal

\begin{tabular}{|c|c|c|c|}
\hline \multirow{3}{*}{ Variables } & \multicolumn{2}{|c|}{ Enfermedad Periodontal } & \multirow{3}{*}{$\mathbf{P}$} \\
\hline & Gingivitis & Periodontitis & \\
\hline & n (\%) & n (\%) & \\
\hline Enjuague bucal & & & 0,567 \\
\hline No & $3(4,7)$ & $61(95,3)$ & \\
\hline $\mathrm{Si}$ & $4(7,1)$ & $52(92,9)$ & \\
\hline Seda dental & & & 0,008 \\
\hline No & $2(2,8)$ & $70(97,2)$ & \\
\hline $\mathrm{Si}$ & $5(10,4)$ & $43(89,6)$ & \\
\hline Palillos & & & 0,006 \\
\hline No & $6(13,6)$ & $38(86,4)$ & \\
\hline $\mathrm{Si}$ & $1(1,3)$ & $75(98,7)$ & \\
\hline Sangrado al cepillado & & & 0,206 \\
\hline No & $4(9,5)$ & $38(90,5)$ & \\
\hline $\mathrm{Si}$ & $3(3,8)$ & $75(96,2)$ & \\
\hline Frecuencia de cepillado & & & 0,300 \\
\hline Una vez & -- & $12(100,0)$ & \\
\hline Dos veces & $3(4,3)$ & $66(95,7)$ & \\
\hline Tres veces & $4(10,3)$ & $35(89,7)$ & \\
\hline Educación en higiene bucal & & & 0,417 \\
\hline No & $2(3,8)$ & $50(96,2)$ & \\
\hline $\mathrm{Si}$ & $5(7,4)$ & $63(92,6)$ & \\
\hline
\end{tabular}

Prevalencia de la enfermedad periodontal según los factores de riesgo de comportamiento y biológico

Para el factor de riesgo tabaquismo, se encontró una diferencia estadísticamente significativa ( $\mathrm{p}=0,007$ ) con un Odds Ratio OR (crudo) de 11,38 [IC 95\% 1,32 - 97,95]. Este valor sugiere que había una probabilidad de 11,38 de presentar enfermedad periodontal entre los fumadores en relación con los soldados que no eran fumadores. Para el grupo de los no fumadores, la prevalencia de periodontitis fue de $86,7 \%$ y de gingivitis del $13,3 \%$ mientras que la prevalencia de periodontitis y de gingivitis en el grupo de fumadores fue $98,7 \% \mathrm{y}$ $1,3 \%$, respectivamente (Tabla 3 ).

Con respecto al consumo de bebidas alcohólicas no se encontró una diferencia estadísticamente significativa $(p=0,478)$, un OR (crudo) de 1,74 [IC $95 \%$ 0,37 - 8,21]. Por tanto, la probabilidad de presentar enfermedad periodontal periodontal entre los consumidores de alcohol fue 1,74 veces en relación con los no consumidores pero es necesario tener en cuenta la no significancia estadística del estimado (Tabla 3). 
Tabla 3. Distribución de factores de riesgo según la enfermedad periodontal

\begin{tabular}{|c|c|c|c|c|c|}
\hline \multirow{3}{*}{ Variables } & \multicolumn{5}{|c|}{ Condición Periodontal } \\
\hline & \multirow{2}{*}{$\begin{array}{c}\text { Gingivitis } \\
\mathbf{n}(\%)\end{array}$} & \multirow{2}{*}{$\begin{array}{c}\text { Periodontitis } \\
\mathbf{n}(\%)\end{array}$} & \multirow{2}{*}{ OR } & \multirow{2}{*}{ IC $95 \%$} & \multirow{2}{*}{$\mathbf{P}$} \\
\hline & & & & & \\
\hline Fuma & & & 11,39 & $1,32-97,95$ & 0,007 \\
\hline $\mathrm{Si}$ & $1(1,3)$ & $74(98,7)$ & & & \\
\hline No & $6(13,3)$ & $39(86,7)$ & & & \\
\hline Consumo bebida alcohólica & & & 1,74 & $0,37-8,21$ & 0,478 \\
\hline $\mathrm{Si}$ & $4(4,8)$ & $79(95,2)$ & & & \\
\hline No & $3(8,1)$ & $34(91,9)$ & & & \\
\hline Placa Bacteriana & & & - & - & -- \\
\hline Niveles mínimos & $7(5,8)$ & - - & & & \\
\hline Niveles > mínimo & - - & $113(94,2)$ & & & \\
\hline
\end{tabular}

\section{Severidad de enfermedad periodontal}

La muestra consistió en 3840 sitios examinados en 120 pacientes, de los cuales el 74,8\% (2872) de los sitios presentaron algún grado de pérdida del nivel de inserción clínica. Se observó que el 36,6\% (1405 sitios) tenía un nivel de pérdida de inserción clínica de 1 a 2 mm; el 27,8\% (1067 sitios) presentó un nivel de pérdida de inserción clínica de 3 a $4 \mathrm{~mm}$ y el 10,5\% (402 sitios) un nivel mayor a $5 \mathrm{~mm}$. De otro lado, el $2,7 \%$ (102 sitios) correspondió al porcentaje de dientes ausentes y el $22,5 \%$ (864 sitios) a zonas sin ninguna pérdida del nivel de inserción clínica (Tabla 4).

Tabla 4. Distribución de la severidad de la enfermedad periodontal.

\begin{tabular}{lc}
\hline Variables & $\mathbf{n}(\%)$ \\
\hline NIC $=0$ & $864(22,5)$ \\
NIC $=1 \mathrm{~mm}-2 \mathrm{~mm}$ & $1405(36,6)$ \\
NIC $=3 \mathrm{~mm}-4 \mathrm{~mm}$ & $1067(27,8)$ \\
NIC $=\geq 6 \mathrm{~mm}$ & $402(10,5)$ \\
Dientes ausentes & $102(2,7)$ \\
Total sitios examinados & $3840(100)$ \\
\hline
\end{tabular}

Con respecto al nivel de inserción clínica de $1 \mathrm{~mm}$ $2 \mathrm{~mm}$ los dientes más afectados fueron los dientes 11 y 15 con un 53,3\%, cada uno, el diente 41 con $52,5 \%$ y los dientes 13 y 42 con un $48,3 \%$ cada uno. Estos valores y los relacionados con la distribución de los sitios según el nivel de inserción clínica se observan en la Tabla 5.

\section{Necesidad de tratamiento según el CPITN}

El 100\% de los sextantes de la población estudiada necesitaban tratamiento. El sextante con el mayor porcentaje con el criterio 4, es decir, que necesitó el tratamiento de mayor complejidad fue el sextante 4 con el 50\%, mientras que el sextante con el criterio 1, esto es "Mejorar la higiene bucal, instrucción en higiene bucal y profilaxis" fue el sextante 2 con un $34,2 \%$ (Tabla 6). De otro lado, en la Tabla 7 se muestran los resultados obtenidos para el CPITN en la población evaluada. 
Tabla 5. Distribución de sitios de acuerdo al Nivel de Inserción Clínica

\begin{tabular}{|c|c|c|c|c|c|}
\hline Diente & $\begin{array}{c}\text { NIC }=0 \\
\text { n (\%) }\end{array}$ & $\begin{array}{c}\text { NIC }=1 \mathrm{~mm}-2 \mathrm{~mm} \\
n(\%)\end{array}$ & $\begin{array}{c}\mathrm{NIC}=3 \mathrm{~mm}-4 \mathrm{~mm} \\
\mathrm{n}(\%)\end{array}$ & $\begin{array}{c}\text { NIC } \geq 5 \mathrm{~mm} \\
\mathrm{n}(\%)\end{array}$ & $\begin{array}{c}\text { Dientes Ausentes } \\
\text { n (\%) }\end{array}$ \\
\hline 18 & $7(5,8)$ & $36(30,0)$ & $47(39,2)$ & $24(20,0)$ & $6(5,0)$ \\
\hline 17 & $12(10)$ & $48(40,0)$ & $40(33,3)$ & $18(15,0)$ & $2(1,7)$ \\
\hline 16 & $7(5,8)$ & $12(10,0)$ & $50(41,7)$ & $40(33,3)$ & $11(9,2)$ \\
\hline 15 & $22(18,3)$ & $64(53,3)$ & $30(25,0)$ & $4(3,3)$ & - \\
\hline 14 & $35(29,2)$ & $53(44,2)$ & $31(25,8)$ & $1(0,8)$ & -- \\
\hline 13 & $45(37,5)$ & $58(48,3)$ & $17(14,2)$ & -. & -- \\
\hline 12 & $60(50,0)$ & $52(43,3)$ & $8(6,7)$ & - & - \\
\hline 11 & $24(20,0)$ & $64(53,3)$ & $32(26,7)$ & - & -- \\
\hline 21 & $63(53,0)$ & $51(43,0)$ & $6(5,0)$ & - & -- \\
\hline 22 & $47(39,2)$ & $53(44,2)$ & $20(16,7)$ & - - & - \\
\hline 23 & $31(25,9)$ & $55(45,8)$ & $34(28,3)$ & -- & -- \\
\hline 24 & $29(24,2)$ & $51(43,0)$ & $39(32,5)$ & $1(0,8)$ & -- \\
\hline 25 & $28(23,3)$ & $49(40,8)$ & $38(31,7)$ & $5(4,2)$ & -- \\
\hline 26 & $7(5,8)$ & $1(0,8)$ & $32(26,7)$ & $66(55,0)$ & $14(11,7)$ \\
\hline 27 & $14(11,7)$ & $41(34,2)$ & $53(44,2)$ & $11(9,2)$ & $1(0,8)$ \\
\hline 28 & $41(34,2)$ & $35(29,2)$ & $36(30,0)$ & $5(4,2)$ & $3(2,5)$ \\
\hline 38 & $25(20,8)$ & $44(36,7)$ & $44(36,7)$ & $4(3,3)$ & $3(2,5)$ \\
\hline 37 & $18(15,0)$ & $54(45,0)$ & $40(33,3)$ & $8(6,7)$ & - - \\
\hline 36 & $7(5,8)$ & $3(1,7)$ & $33(27,5)$ & $60(50,0)$ & $18(15,0)$ \\
\hline 35 & $17(14,1)$ & $57(48,0)$ & $44(36,7)$ & $2(1,7)$ & - \\
\hline 34 & 20 (16.7) & $56(46,7)$ & $43(35,8)$ & $1(0,8)$ & -- \\
\hline 33 & $39(33,0)$ & $45(38,0)$ & $34(28,3)$ & $2(1,7)$ & -- \\
\hline 32 & $47(39,2)$ & $63(53,0)$ & $10(8,3)$ & - & -- \\
\hline 31 & $7(5,8)$ & $15(13,0)$ & $53(44,2)$ & $42(35,0)$ & $3(2,5)$ \\
\hline 41 & $50(41,7)$ & $63(52,5)$ & $7(5,8)$ & - - & - - \\
\hline 42 & $45(38,0)$ & $58(48,3)$ & $15(12,5)$ & $2(1,7)$ & - \\
\hline 43 & $46(38,3)$ & $54(45,0)$ & $19(15,8)$ & $1(0,8)$ & -- \\
\hline 44 & $30(25,0)$ & $50(41,7)$ & $39(32,5)$ & $1(0,8)$ & -- \\
\hline 45 & $17(14,2)$ & $41(34,2)$ & $54(45,0)$ & $8(6,7)$ & - \\
\hline 46 & $7(5,8)$ & $2(1,7)$ & $7(5,8)$ & $77(64,2)$ & $29(24,2)$ \\
\hline 47 & $10(8,3)$ & $38(31,7)$ & $53(44,2)$ & $16(13,3)$ & $3(2,5)$ \\
\hline 48 & $10(8,3)$ & $39(33,0)$ & $59(49,2)$ & $3(3,0)$ & $9(7,5)$ \\
\hline
\end{tabular}

Tabla 6. Distribución de los sextantes según marcadores clínicos del CPITN

\begin{tabular}{cccccc}
\hline & \multicolumn{5}{c}{ Criterios CPITN } \\
\cline { 2 - 6 } Sextantes & $\mathbf{0}$ & $\mathbf{1}$ & $\mathbf{2}$ & $\mathbf{3}$ & $\mathbf{4}$ \\
\cline { 2 - 6 } & $\mathbf{n ~ ( \% )}$ & $\mathbf{n ~ ( \% )}$ & $\mathbf{n ~ ( \% )}$ & $\mathbf{n}(\%)$ & $\mathbf{n}(\%)$ \\
\hline Sextante 1 & -- & $7(5,8)$ & $12(10,0)$ & $48(40,0)$ & $53(44,2)$ \\
Sextante 2 & -- & $41(34,2)$ & $36(30,0)$ & $30(25,0)$ & $13(10,8)$ \\
Sextante 3 & -- & $7(5,8)$ & $20(16,7)$ & $49(40,8)$ & $44(36,7)$ \\
Sextante 4 & -- & $10(8,3)$ & $13(10,8)$ & $37(30,8)$ & $60(50,0)$ \\
Sextante 5 & -- & $27(22,5)$ & $45(37,5)$ & $28(23,3)$ & $20(16,7)$ \\
Sextante 6 & -- & $7(5,8)$ & $14(11,6)$ & $57(47,5)$ & $42(35,0)$ \\
\hline
\end{tabular}

0: no necesita tratamiento; 1: mejorar la higiene, profilaxis; 2: instrucción higiene bucal, profilaxis, detartraje; 3: instrucción en higiene bucal, alisado radicular a campo cerrado; 4: 1 y 2 , alisado radicular a campo abierto. 
Tabla 7. Distribución del Índice Comunitario de Necesidad de Tratamiento Periodontal en el personal del Batallón de Artillería y Defensa Aérea No. 2 Nueva Granada

\begin{tabular}{llc}
\hline \multicolumn{1}{c}{ Variables } & \multicolumn{1}{c}{ Necesidad de Tratamiento } & $\%$ \\
\hline Criterio 0 - Periodonto sano & No necesita tratamiento, mantener medidas de prevención & - \\
Criterio 1 - Sangrado al sondaje & $\begin{array}{l}\text { Mejorar la higiene bucal, instrucción en higiene bucal y profilaxis } \\
\text { Instrucción en higiene bucal, profilaxis, eliminar obturaciones } \\
\text { Criterio } 2 \text { - Cálculo }\end{array}$ & $\begin{array}{l}\text { defectuosas, detartraje } \\
\text { Criterio } 3 \text { - Bolsas de } 4 \text { a } 5 \mathrm{~mm}\end{array}$ \\
$\begin{array}{l}\text { Instrucción en higiene bucal, profilaxis, alisado radicular a campo } \\
\text { Cerrado }\end{array}$ & 34,6 \\
Criterio 4 - Bolsas $\geq 6 \mathrm{~mm}$. & Criterio 1 y 2, alisado radicular a campo abierto & 32,2 \\
\hline
\end{tabular}

\section{DISCUSIÓN}

La prevalencia de la enfermedad periodontal observada fue del 100\%; estos resultados son similares a los reportados por Orozco y colaboradores donde el $81 \%$ de los habitantes de una población aislada de Colombia estaba afectada periodontalmente. ${ }^{6}$ Cabe señalar que estos dos estudios tienen en común que las poblaciones tienen hábitos y condiciones de vida similares, además de no contar con acceso a servicio odontológico. Los resultados de la Tercera Encuesta de Salud Bucal (ENSAB III) realizada en Colombia evidenciaron una prevalencia de 50,2\% para todas las formas de enfermedad periodontal (gingivitis y periodontitis). ${ }^{7}$ De igual manera los resultados de un estudio realizado en la facultad de odontología de la Universidad Nacional Autónoma de México mostró un prevalencia del $67,2 \%$ para esta enfermedad. ${ }^{8}$

Según las variables demográficas y socioeconómicas, la prevalencia más alta de periodontitis la presentó la población con el nivel de educación secundaria con un $90,7 \%$ y la población que pertenecía al estrato socioeconómico 3 con un 95,8\%. Estos resultados difieren con los encontrados por Katz y colaboradores en una población militar israelí donde los individuos con un nivel de educación ( \pm 12 años) tenían menos sangrado y mejores condiciones económicas que los individuos con menos de 12 años de educación. ${ }^{9}$ Así mismo, el estudio realizado por Vered y colaboradores en jóvenes militares israelíes mostró que las personas que completaron doce años de escolaridad eran más propensos a estar sanos que los que tenían una nivel de educación inferior. ${ }^{10}$ Dye y Vargas analizaron un gran número de habitantes norteamericanos y demostraron que los grupos de bajo nivel educativo o en desventaja social, necesitaban instrucción en higiene bucal en el $90 \%$, debido a que era significativa la desigualdad de las necesidades de tratamiento periodontal con un consecuente aumento en la complejidad terapéutica. ${ }^{11}$ Los anteriores estudios sugieren que la prevalencia de la enfermedad periodontal se acentúa más en las personas con menor grado de educación y una condición socioeconómica más baja.

De acuerdo con las prácticas, uso de elementos e instrucción en higiene oral se presentó una mayor prevalencia de enfermedad en los soldados que no utilizaban enjuague bucal ni seda dental. Así mismo, la prevalencia más alta se observó en el personal que manifestó sangrar durante el cepillado, se cepillaban dos veces al día y no habían recibido educación en higiene oral. Un estudio realizado en cadetes de la marina en el sur de la India reveló que más de la mitad de éstos $(54,6 \%)$ sabía que el cepillado de dientes ayudaba en la prevención de la caries dental y el $42,2 \%$ era consciente de que el cepillado prevenía enfermedades de las encías. ${ }^{12}$ Un número considerable $(66,3 \%)$ no era consciente de que la seda dental fuera útil en la prevención de la caries dental y las enfermedades de las encías, mientras que 33,7\% de los cadetes sabía que ésta sí ayudaba a prevenirlas. Otero y Proaño en su estudio en el personal de tropa masculino en servicio militar de Lima (Perú) encontraron que el 63\% de la población manifestó cepillarse los dientes dos veces al día y que el $96,6 \%$ no usaba seda dental y para tales fines utilizan hilo de coser, palillos, palitos de fósforo y sal. ${ }^{13}$ Estos estudios sugieren que la enfermedad periodontal independientemente de la ubicación geográfica prevalece más en las poblaciones que no poseen los conocimientos sobre el cuidado de la salud oral, no practican una buena higiene y no tienen una buena actitud de autocuidado.

Se considera que la enfermedad periodontal tiene múltiples factores de riesgo que constituyen una parte de la cadena etiológica de la enfermedad. Para la realización de este estudio, se tuvo en cuenta personas fumadoras, el consumo de bebidas alcohólicas y los niveles de placa bacteriana. Es así como los resultados mostraron una alta prevalencia de periodontitis para los fumadores de $98,7 \%$. 
Estudios realizados por Bimstein y colaboradores mostraron que la enfermedad periodontal es más prevalente en la población adulta sin embargo, también es común y de importancia entre los adultos jóvenes, adolescentes e incluso en los niños. ${ }^{14} \mathrm{Al}-$ gunos autores han sugerido que el efecto de fumar entre las poblaciones más jóvenes es similar que entre las personas mayores con el agravante que en los primeros se duplicará la probabilidad de enfermedad periodontal en la etapa adulta si se empieza este hábito desde una edad temprana o a mediados de la adolescencia. ${ }^{15,16}$

En el presente estudio, se observó que los consumidores de bebidas alcohólicas tenían una prevalencia de periodontitis de 95,2\%. En una revisión sistemática realizada por Amaral y colaboradores encontraron diferentes hallazgos en los estudios seleccionados: ${ }^{17}$ concluyen que hay pruebas sólidas que sugieren que el alcohol es un indicador de riesgo para la periodontitis y sugieren realizar más estudios longitudinales sobre la asociación de la dependencia y el consumo del alcohol con la enfermedad periodontal.

Este estudio confirmó una vez más que los altos niveles de placa bacteriana están relacionados directamente con la prevalencia de la enfermedad. Tal como lo revela en su revisión, Newman dice: "La naturaleza de la relación entre la placa dental y la enfermedad periodontal sigue siendo poco clara, aunque no hay duda de que la placa es la causa directa". ${ }^{18}$ Gonzáles y colaboradores demostraron una dependencia altamente significativa con una relación inversamente proporcional entre la higiene bucal, y la prevalencia y gravedad de la enfermedad periodontal. ${ }^{19}$ Estos resultados sugieren que la placa bacteriana sigue siendo un factor de riesgo para el desarrollo de la enfermedad periodontal, es necesaria la presencia de ciertas bacterias para que ocurra la periodontitis aunque no es causa suficiente pues depende de la respuesta del huésped, esto explica porque muchos individuos pueden albergar los microorganismos y no desarrollar la enfermedad.

De otro lado, la pérdida del nivel de inserción clínica ocupó el $74,8 \%$ de los sitios examinados mientras que otros autores han reportado cifras de $67,9 \%$ y $55,7 \%{ }^{8,20}$ Las necesidades de tratamiento de acuerdo al CPITN arrojaron resultados equiparables con los obtenidos por Doncel y Castillo donde el 67,6\% de su población tenía afección periodontal y requerían tratamiento. ${ }^{21}$ En el estudio de García-Conde y colaboradores, se encontró que el $94 \%$ de la población necesitaba tratamiento periodontal. De igual manera, en Argentina el $96,8 \%$ de los individuos participantes en un estudio epidemiológico nacional requerían algún tipo de tratamiento y en este caso el $100 \%$ de la población evaluada lo necesitaba. 22,23

La distribución de todas estas condiciones periodontales demuestran la elevada prevalencia de la enfermedad periodontal así como sus factores de riesgo y severidad en diferentes puntos geográficos y en diferentes poblaciones. En este sentido, se concluye que la prevalencia de enfermedad periodontal en este grupo poblacional fue muy alta (100\%). Sin embargo, no se encontró una relación entre el nivel de escolaridad, el estrato socioeconómico y la tendencia de enfermedad periodontal.

La población objeto de este estudio necesita acceder a educación en higiene oral para mejorar sus hábitos de higiene y usar correctamente los elementos para lograr su salud periodontal. La interacción frecuente de factores de riesgo biológicos y de comportamiento contribuyen al aumento de la prevalencia, severidad de la enfermedad y al impacto que tiene ésta sobre la calidad de vida, aunque este aspecto debe ser estudiado a profundidad.

Las repercusiones de no conocer el estado de salud periodontal y la necesidad de tratamiento de los soldados puede afectar la buena marcha del estamento militar, inferir en la participación activa del personal en el cumplimiento de su servicio debido a una emergencia de origen periodontal que amerite recursos para su tratamiento. Por tal motivo, se sugiere a los profesionales del área odontológica que brindan atención al personal militar, genere estrategias que permitan el mejoramiento de los protocolos de atención, tratamientos preventivos y remisiones oportunas al periodoncista para diagnosticar y tratar a tiempo la enfermedad.

\section{Agradecimientos}

Los autores desean expresar un especial agradecimiento al señor comandante del Batallón de Artillería de Defensa Aérea No. 2 Nueva Granada, Teniente Coronel Jaime H. Pongutá S., por su invaluable apoyo para la realización de este proyecto.

\section{BIBLIOGRAFÍA}

1. Botero JE, Bedoya E. Determinantes del diagnóstico periodontal. Rev Clin Periodoncia Implantol Rehabil Oral 2010; 3 (2): $94-99$.

2. Informe de la Organización Mundial de la Salud sobre enfermedades bucodentales. [en línea]. 2004; [Fecha de acceso: febrero de 2013]. URL disponible en: http://www. who.int/mediacentre/news/releases/2004/pr15/es/

3. García-Conde GG, Espinosa de Santillana IA, MartínezArroniz F, Huerta-Herrera N, Islas-Márquez AJ, Medina-Solís $C E$. Necesidades de tratamiento periodontal en adultos de la región rural Mixteca del Estado de Puebla, México. Rev Salud Pública [en línea] 2010; 12 (4). URL disponible en: http://www.scielosp.org/scielo.php?script=sci_arttext\&pid $=$ S0124-00642010000400011 
4. Alvear FS, Vélez ME, Botero L. Factores de riesgo para las enfermedades periodontales. Rev Fac Odontol Univ Antioq 2010; 22 (1): 109 - 111.

5. Dirección General de Sanidad Militar. Fuerzas Militares de Colombia, Comando General de las fuerzas Militares. Directiva Transitoria No.142614 CGFM--DGSM-SS-GSP-23.1. [en línea]. 2010. [Fecha de acceso: octubre de 2012]. [Aprox.1 p] URL disponible en:http://www.sanidadfuerzasmilitares.mil.co/la_entidad/normatividad/directivas/ directiva_transitoria_142614_2010c.

6. Orozco AH, Franco AM, Ramírez-Yánez GO. Periodontal treatment needs in a native island community in Colombia determined with CPTIN. Int Dent J 2004; 54 (2): 73 - 76.

7. República de Colombia, Ministerio de Salud. III Estudio Nacional de Salud Bucal - ENSAB III .II Estudio Nacional de Factores de Riesgo de Enfermedades Crónicas - ENFREC II.

8. Rojo NR, Flores A, Arcos M. Prevalencia, severidad y extensión de periodontitis crónica. Revista Odontológica Mexicana 2011; 15 (1): 31 - 39.

9. Katz J, Peretz B, Sgan-Cohen HD, Horev T, Eldad A. Periodontal status by CPITN, and associated variables in an Israeli permanent force military population. J Clin Periodontol 2000; 27 (5): 319 - 324.

10. Vered Y, Livny A, Zini A, Sgan-Cohen HD. Periodontal health status and smoking among young adults. J Clin Periodontol 2008; 35 (9): 768 - 772.

11. Dye BA, Vargas CM. The use of a modified CPITN approach to estimate periodontal treatment needs among adults aged 20-79 year by socio-demographic characteristics in the United States, 1988-94. Community Dent Health 2002; 19 (4): 215 - 223.

12. Singh A. Oral health knowledge, attitude and practice among NCC Navy Cadets and their correlation with oral hygiene in south India. Oral Health Prev Dent 2009; 7 (4): $363-367$.

13. Otero J, Proaño D. Prevalencia de enfermedades periodontales, factores de riesgo y necesidad de tratamiento en el personal de tropa masculino en Servicio Militar en Lima en el año 2000. Rev Estomatol Herediana 2005; 15 (1): $11-17$.

14. Bimstein E, Needleman HL, Karimbux N,Van Dyke TE. Periodontal and Gingival Health and Diseases: Children, Adolescents, and Young Adults. London: Martin Dunitz Ltd; 2001. p 3 -16.

15. Machuca G, Rosales I, Lacalle JR, Machuca C, Bullon P. Effect of cigarette smoking on periodontal status of healthy young adults. J Periodontol 2000; 71 (1): 73 - 78.

16. Hashim R, Thomson W, Pack AR. Smoking in adolescence as a predictor of early loss of periodontal attachment. Community Dent Oral Epidemiol 2001; 29 (2): 130 - 135.

17. Amaral C, Vettore M, Leão A. The relationship of alcohol dependence and alcohol consumption with periodontitis: A systematic review. J Dent 2009; 37 (9): 643 - 651.

18. Newman HN. Plaque and chronic inflammatory periodontal disease. A question of ecology. J Clin Periodontol 1990; 17 (8): 533 - 541.

19. González ME, Toledo B, Nazco C. Enfermedad periodontal y factores locales y sistémicos asociados. Rev Cubana Estomatol [en línea] 2002 [Fecha de acceso: abril de 2013]; 39 (3). URL disponible en:http://scielo.sld.cu/scielo.php?pid = S0034-75072002000300006\&script = sci_arttext

20. Holmberg F, Zaror C, Mendez F, Holmberg F, Holmberg H. Comparación del nivel de inserción periodontal entre in- dividuos fumadores y no fumadores. Int J Odontostomat 2008; 2 (2): 175 - 181.

21. Doncel C, Castillo A. Determinación de la necesidad de tratamiento periodontal en jóvenes de una escuela militar. Rev Cub Med Mil [en línea] 2008 [Fecha de acceso: abril de 2013]; 37 (1). URL disponible en: http://scielo.sld.cu/scielo.php?script = sci_arttext\&pid $=$ S0138-65572008000100004

22. García-Conde G, Espinosa IA, Martínez-Arroniz F, HuertaHerrera N, Islas-Márquez A, Medina-Solís CE. Necesidades de tratamiento periodontal en adultos de la región rural Mixteca del Estado de Puebla, México. Rev Salud Pública 2010; 12 (4): 647 - 657

23. Estudio Epidemiológico Nacional: Diagnóstico de la Necesidad de Tratamiento Periodontal en Adultos de la República Argentina. Dirección y Coordinación - Sociedad Argentina de Periodontología (Seccional Asociación Odontológica Argentina) - Confederación Odontológica de la República Argentina (Departamento de Educación para la Salud).

\section{Correos electrónicos de los autores:}

Claudia Liliana Álvarez Cortés: clalvarez_3@hotmail.com Vietnamila Rico Jaimes: vietnamila@yahoo.com Juan Carlos Gómez Herrera: juankarlos60@gmail.com 